\title{
Deteç̧ão de Anomalias em Comportamento de Abelhas Utilizando Redes Neurais Recorrentes
}

\author{
Hanna V. F. de Carvalho ${ }^{1}$, Eduardo C. Carvalho ${ }^{1}$, Helder Arruda ${ }^{3}$, \\ Vera Imperatriz-Fonseca ${ }^{3}$, Paulo de Souza $^{2}$, Gustavo Pessin ${ }^{3}$ \\ ${ }^{1}$ Instituto de Ciências Exatas e Naturais \\ Universidade Federal do Pará (UFPA) - Belém, PA, Brasil \\ ${ }^{2}$ Data61, CSIRO - Sandy Bay, Tasmânia, Austrália \\ ${ }^{3}$ Instituto Tecnológico Vale - Belém, PA, Brasil \\ hanna.carvalhogepq.itv.org
}

\begin{abstract}
Resumo. Abelhas tem um papel muito importante na polinização de espécies vegetais. Por meio da polinização, as espécies vegetais trocam gametas e tem frutos em maior quantidade e em melhor qualidade. Conhecer o comportamento das abelhas é importante pois auxilia produtores a entender melhor as atividades das abelhas relacionadas com a polinização. Neste trabalho avaliamos como técnicas de aprendizado de máquina podem ser empregadas na tarefa de identificação de anomalias em comportamento de abelhas. Mais de 1200 abelhas de oito colmeias foram monitoradas com etiquetas eletrônicas (RFIDs) num período de 4 semanas. Mostramos como diferentes topologias de redes recorrentes podem auxiliar na tarefa de classificação de anomalia comportamental.
\end{abstract}

\section{Introdução}

A primeira grande importância das abelhas sociais para o homem refere-se à utilização do mel produzido por estas, que se tornou de grande valor comercial, assim como a cera e a resina. Esses produtos tornaram-se ainda de grande importância para a complementação do comércio rural da região Norte, assim como foi demonstrado no trabalho de [Venturieri et al. 2003]. Hoje, sabe-se que as abelhas desempenham um papel fundamental para manutenção da biodiversidade, por meio da polinização, sendo consideradas os agentes polinizadores mais eficientes para o processo. Imperatriz-Fonseca, Contrera e Kleinert [Imperatriz-Fonseca et al. 2004] descrevem que o valor anual estimado gerado pela atividade das abelhas para polinização, foi de US\$65-70 bilhões por ano. Mais de $80 \%$ de todas as espécies de plantas com flores e mais de 3/4 das culturas agrícolas do mundo dependem de animais polinizadores. A maioria delas pode ser polinizada por uma ou mais entre as 20.000 espécies de abelhas conhecidas. Temos ainda culturas, como a do maracujá, em que é utilizada a polinização manual, mas as abelhas substituem com vantagem este trabalho caro e árduo; os frutos resultantes apresentam melhor qualidade e sabor.

Apesar do crescimento global do número de colmeias domesticadas, a quantidade de abelhas vem diminuindo nos Estados Unidos desde a década de quarenta e em alguns países da Europa desde a década de sessenta [Potts et al. 2010]. Essa diminuição afeta não só as plantas oriundas das florestas nativas, mas também tem um impacto 
significativo na agricultura e, consequentemente, na economia. Portanto, estudar esses insetos e suas atividades tornam-se de extrema importância na atualidade. Infelizmente, há algumas poucas evidências que tentam justificar este desaparecimento. Alguns estudos indicam que esse cenário está relacionado a um conjunto de fatores como o surgimento de novos tipos de parasitas, utilização de pesticidas, cultivo de monoculturas, ondas eletromagnéticas geradas por torre de telefonia celular, vegetação alterada geneticamente e o manejo inadequado de colmeias [Message et al. 2012].

Percebemos então, a grande importância de tentar encontrar dados anômalos na atividade média das abelhas, que poderiam representar empecilhos e problemas para o desenvolvimento e desempenho dessas, como agentes polinizadores. Conhecer melhor o comportamento desses insetos pode beneficiar apicultores, agricultores e pesquisadores a tomar medidas para melhorar e ajudar no processo de polinização, maximizando a produção de frutos com melhor qualidade. Além de enriquecer os conhecimentos e informações sobre o comportamento das abelhas. Trabalhos recentes utilizam técnicas de detecção de anomalias, que se trata do processo de encontrar padrões em dados que não estejam de acordo com um comportamento a priori esperado, ou seja, um problema em que algumas amostras estão distantes, de acordo com determinada métrica, do resto do conjunto de dados, e então essas amostras anômalas são descritas como outliers. Atualmente, técnicas de detecção de anomalias estão sendo bastante difundidas devido à aplicabilidade em problemas reais, como detecção de fraudes, de erros de coleta de dados de sensores, de falhas em sistemas de monitoramento de saúde, etc.

As Redes Neurais Recorrentes são uma técnica de aprendizado de máquina que apresentam neurônios recorrentes. Como estas incluem loops, elas podem armazenar informações ao processar novas entradas. Portanto, a memória as torna ideais para tarefas de processamento de dados que utilizam séries temporais. Este trabalho investiga a capacidade das redes neurais propostas em classificar o comportamento de abelhas monitoradas através de etiquetas eletrônicas. Neste estudo foram utilizadas abelhas da espécie Melipona fasciculata (uruçu cinzenta), que são responsáveis pela polinizações de muitas culturas e com alto valor de produção. A importância dos meliponíneos vai muito além dos benefícios econômicos, por exemplo, na reconstituição de florestas tropicais e conservação dos remanescentes [Silva and da Paz 2012]. Estas abelhas atuam também como bioindicadoras da qualidade ambiental [P. Ballivian 2008]. Apesar disso, as espécies de meliponíneos encontram-se em processo acelerado de desaparecimento, provocado principalmente pela perda de habitats e desmatamento de florestas nativas, ambiente preferencial dessas espécies [Lopes et al. 2005]. Nosso objetivo é investigar uma metodologia de aprendizado de máquina para detecção de anomalias, testando diferentes arquiteturas de Redes Neurais para a tarefa de classificação de eventos anômalos na atividade de abelhas monitoradas através de etiquetas Radio-Frequency IDentification (RFID).

Este trabalho está dividido da seguinte forma: No Capítulo 2 são apresentados conceitos empregados ao longo deste trabalho, como aprendizado de máquina, redes neurais, redes LSTM e detecção de anomalias. No Capítulo 3 são apresentados trabalhos atuais relacionados à detecção de anomalias. O Capítulo 4 apresenta a metodologia utilizada na confecção do trabalho, assim como a coleta e processamento de dados, e os modelos propostos. O Capítulo 5 apresenta os resultados obtidos. Finalizaremos o documento com o Capítulo 6, apresentando as considerações finais e os trabalhos futuros. 


\section{Referencial Teórico e Conceitos Gerais}

\subsection{Séries Temporais}

Segundo Wooldridge [Wooldridge 2012], uma série temporal pode ser descrita como uma sequência de realizações (observações) de uma variável ao longo do tempo, ou seja, é uma sequência de números coletados em intervalos regulares durante um período de tempo, que representam características quantitativas de um fenômeno individual ou coletivo em períodos sucessivos, e geralmente, equidistantes, dos períodos de tempo. As séries temporais tem sido bastante utilizadas, em aplicações reais como: previsão econômica, previsão de vendas, análise orçamentária, análise do mercado de ações, projeções de rendimento, etc. E no caso deste trabalho, utilizamos uma série temporal de coletas de atividade média de abelhas para classificação.

\subsection{Aprendizado de Máquina}

Aprendizado de Máquina é uma área da Inteligência Artificial que tem como objetivo desenvolver técnicas computacionais de aprendizado e de aquisição de conhecimentos [Mitchell 1997], [Baranauskas and Monard 2003]. Essas técnicas devem exibir comportamento inteligente e realizar tarefas complexas com um nível de competência equivalente ou superior ao de um especialista humano [Nikolopoulos 1997]. Das diversas técnicas de ML existentes, pode-se dizer que a grande maioria tem foco em grupos relativamente específicos de aplicações. É difícil imaginar uma técnica que seja capaz de resolver todas as gamas de problemas que envolvam aprendizado automático, seja ele supervisionado, não supervisionado ou semi-supervisionado [Mitchell 1997].

As redes Multilayer Perceptron podem ser consideradas as Redes Neurais Artificiais mais populares, conhecida também como redes feedforward. A unidade fundamental de processamento de informação de uma RNA é o neurônio artificial, que possui três principais elementos: os pesos sinápticos, um somador e a função de ativação (Figura $1(\mathrm{a})$ ).

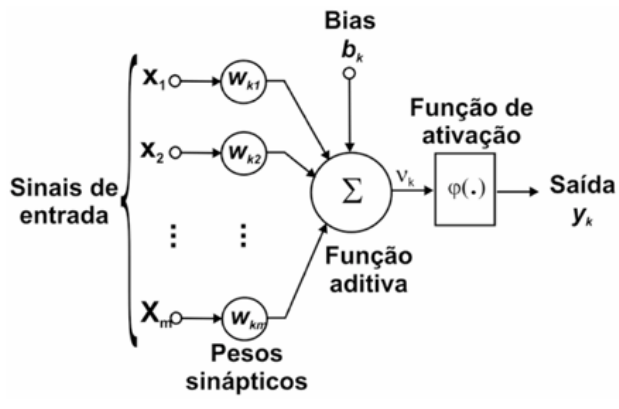

(a)

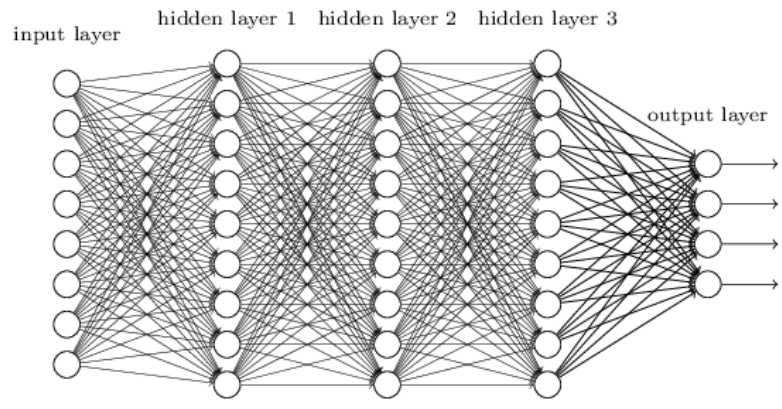

(b)

Figura 1. (a) Representação de um neurônio artificial. (b) Exemplo de MLP.

As Redes Neurais Recorrentes são um caso especial de RNAs onde existe realimentação de entradas [Segatto and Coury 2006], isto faz com que apresentem comportamento dinâmico temporal. Por apresentarem capacidade de memória, são muito empregadas em tarefas relacionadas a séries temporais. Enquanto redes com conexões padrão respondem a uma dada entrada sempre com a mesma saída, uma rede recorrente pode responder à mesma entrada com diferentes saídas em diferentes momentos, dependendo da 
entrada que foi apresentada anteriormente [Penna 2004]. O maior interesse em redes neurais recorrentes está no seu processamento temporal e na sua capacidade de implementar memórias adaptativas [Bengio et al. 1970]. As redes Long Short-Term Memory (LSTM) são um tipo especial de RNN, capaz de aprender informações de longo prazo. Eles foram introduzidos por Hochreiter e Schmidhuber [Hochreiter and Schmidhuber 1997] e foram aprimoradas em muitos outros trabalhos. As redes neurais recorrentes simples são capazes de interligar informações anteriores à tarefa atual, porém na prática, quando o conjunto de dados é muito grande, e as informaçoõs anteriores tornam-se de longo prazo, as RNNs não apresentam a capacidade de aprendizado esperada. As LSTMs são amplamente utilizadas para esses problemas de dependência de longo prazo.

\subsection{Detecção de Anomalias}

De acordo com Hawkins [Hawkins 1980] uma anomalia (outlier) é uma observação que se desvia muito em relação a outras observações que levam a suspeitas de serem geradas por algum mecanismo diferente. Detectar anomalias consiste na identificação de padrões em dados com um comportamento diferente do que seria esperado, um ponto que é muito diferente dos outros. Assim, a detecção de anomalia objetiva encontrar padrões em um conjunto de dados que não estão em conformidade com uma definição conhecida como comportamento normal [Singh and Upadhyaya 2012]. Técnicas de detecção de anomalias podem ser empregadas em diversas áreas, se tornando útil para detecção de fraude, na área de saúde, detecção de intrusos em um sistema, investigação criminal, detecção de erros de medição em dados de sensores, etc. [Gama et al. 2017]. Em cada uma dessas aplicações existe uma maneira para lidar com as classificações de eventos anômalos ou normais. Ou seja, não existe um único método para detecção de anomalia, mas sim um conjunto de abordagens adequadas para os diversos domínios.

Tipos de anomalias podem ser classificados da seguinte forma: (1) Anomalia pontual: Dados individuais que se apresentam muito distantes do restante da distribuição dos outros dados. Consideranto, portanto, pontos que estão distantes de um grupo de pontos, como anomalias. (2) Anomalia contextual: A instância é considerada anômala para determinada condição ou contexto, mas não necessariamente em outro. Para definir as instâncias de dados, utilizam-se atributos contextuais (utilizados para determinar o contexto da instância. Por exemplo, a mesma temperatura ocorrer em estações diferentes do ano, para certa estação, temperaturas baixas são normais, para outra, anomalias. ou atributos comportamentais: Definem as características não-contextuais de uma instância. (3) Anomalia coletiva: Um ponto individual pode não representar uma anomalia, mas quando se considera que todo um conjunto de dados apresenta discrepância em relação ao restante do conjunto de dados, considera-se este tipo de anomalia.

As técnicas de detecção de anomalias podem ser: (1) Supervisionada: Necessita que o conjunto de dados já esteja rotulado para as classes normal e anômala. (2) Semi-Supervisionada: Assume-se que os dados de treino só têm tipos de dados da classe normal. (3) Não-Supervisionada: Não utilizam dados de treinamento. Detecta-se a anomalia no conjunto de teste não-rotulado, partindo do pressuposto que os dados normais são muito mais frequentes do que as anomalias, procurando por instâncias que parecem ser discrepantes do restante do conjunto de dados. Neste trabalho, utilizamos a detecção supervisionada, visto que os dados foram rotulados previamente como sendo anômalos ou normais. 


\section{Trabalhos Relacionados}

Gama et. al. [Gama et al. 2017] apresenta metodologias que combinavam métodos não supervisionados para detecção de anomalia baseado em distância e modelos supervisionados baseados em aprendizado de máquina. Os resultados mostraram que a combinação das técnicas permite a detecção de eventos anômalos em comportamento de insetos de forma satisfatória. Um algoritmo de detecção não supervisionado foi empregado para validar a rotulagem de dados chamado Local Outlier Factor.

Odoux et. al. [Odoux et al. 2014], apresenta o ECOBEE, um esquema de monitoramento de colônias destinado especificamente a fornecer apicultores e pesquisadores, dados ecológicos básicos sobre abelhas em agrossistemas intensivos, bem como a dinâmica da população de colônias. O ECOBEE foi lançado em 2008 como um projeto ecológico a longo prazo com três objetivos específicos: 1. monitorar parâmetros dinâmicos da população sazonal e interanual das colônias de abelhas em um sistema agrícola heterogêneo; 2 . fornecer conjuntos de dados relevantes e robustos para testar hipóteses específicas sobre abelhas, como a influência do planejamento paisagístico, insumos agrícolas ou pressão humana; e 3. oferecer oportunidades para avaliar a eficácia dos esquemas agro-ambientais ou os efeitos das mudanças nas políticas agrícolas sobre o bem-estar das abelhas. Zacepins et. al. [Zacepins et al. 2015], apresenta desafios no desenvolvimento de Precision Beekeeping (PB), que é uma estratégia de gestão apícola baseada no monitoramento de colônias de abelhas individuais para minimizar o consumo de recursos e maximizar a produtividade das abelhas. Apontam ainda que os sistemas PB devem ser otimizados pela busca de combinações apropriadas de diferentes sensores, e os sistemas de suporte à decisão correspondentes devem fornecer soluções convenientes, confiáveis e econômicas. O desenvolvimento e a especificação dos sistemas PB devem considerar interesses comerciais, distância ao apiário, riscos esperados e outras peculiaridades.

Em seu trabalho Application of wireless sensor networks for beehive monitoring and in-hive thermal patterns detection, Kridi, Carvalho e Gomes [Kridi et al. 2016], utilizaram padrões térmicos como um parâmetro para alertar sobre problemas de superaquecimento dentro das colmeias, e isso é importante porque as altas temperaturas podem causar muitos danos à apicultura, como absconding (um processo biológico em que todas as abelhas deixam o ninho estabelecido para buscar um novo adequado), malformação e morte da prole e até mesmo a má qualidade do mel. Os três objetivos ressaltados deste artigo são: (i) obter dados suficientes para a temperatura interna e externa da colméia; (ii) usando técnicas de agrupamento de similaridade para descobrir padrões nos conjuntos de dados mencionados; e (iii) aplicar e testar padrões obtidos em relação às leituras usando Wireless Sensor Network (WSN), em cenários normais e de aquecimento. Ainda, existem trabalhos como o de Salem et. al. [Salem et al. 2014], que propõe uma abordagem leve para a detecção online de medições defeituosas, analisando os dados de saúde coletados de redes sem fio a partir de sensores adaptados ou implantados no corpo de pacientes. O framework proposto realiza análise de dados seqüencial usando um smartphone como estação base, levando em consideração seus recursos restritos, como poder de processamento e capacidade de armazenamento. O objetivo principal do trabalho é criar alarmes somente quando os pacientes entram em uma situação de emergência e descartar falsos alarmes desencadeados por medições defeituosas ou sensores problemáticos.

$\mathrm{Na}$ indústria petrolífera, a detecção de outliers também se faz presente. Martí 
et. al. [Marti et al. 2015] utiliza dados de centenas de sensores presentes em cada uma das máquinas de extração pesada de petróleo para operações de bombeamento e geração, (como turbomáquinas) que enviam dados em alta frequiência para prevenção de danos. Possuir esse tipo de informações ajudaria um operador do sistema a tomar decisões para evitar, corrigir ou reagir às situações associadas à prevenção de danos nas máquinas corretamente. Portanto, os autores propõem a combinação de um algoritmo de segmentação (YASA) rápido e de alta qualidade juntamente com uma abordagem de SVM de classe única para deteção eficiente de anomalias.

\section{Metodologia}

O processo de coleta foi realizado através de um sistema instrumentado conforme mostra a figura 2. A espécie de abelha Melipona fasciculata foi analisada, junto à Embrapa Amazônia Oriental (Belém-PA-BR). No canto inferior a presença da abelha (M. fasciculata nativa da região amazônica) com etiqueta de RFID presa junto ao tórax. O período de coleta foi entre 1 a 31 de Agosto de 2015 onde afixamos etiquetas eletrônicas RFID (Radio-Frequency Identification) em um total de 1280 abelhas sendo 40 abelhas por colmeia a cada semana. O sistema instrumentado detecta o momento que uma abelha passa pelo leitor RFID e, neste momento, registra-se um movimento. Neste período registrou-se um total de 127.758 atividades o que significa aproximadamente 100 registros de atividade por abelha foram detectados durante todo o experimento.

Como vimos, para calcular o nível de atividade das abelhas no sistema basta dividirmos o número total de movimentos em um dado período, pelo número de abelhas vivas naquele instante. Para ilustrar, considere que quando o valor do nível de atividade for 0.0 significa que nenhuma abelha está realizando atividade naquele momento se, por exemplo, for 1.0 significa dizer que cada abelha está realizando em média uma única atividade naquele momento. Durante o experimento havia em média, entre 240 e 320 abelhas vivas por dia.
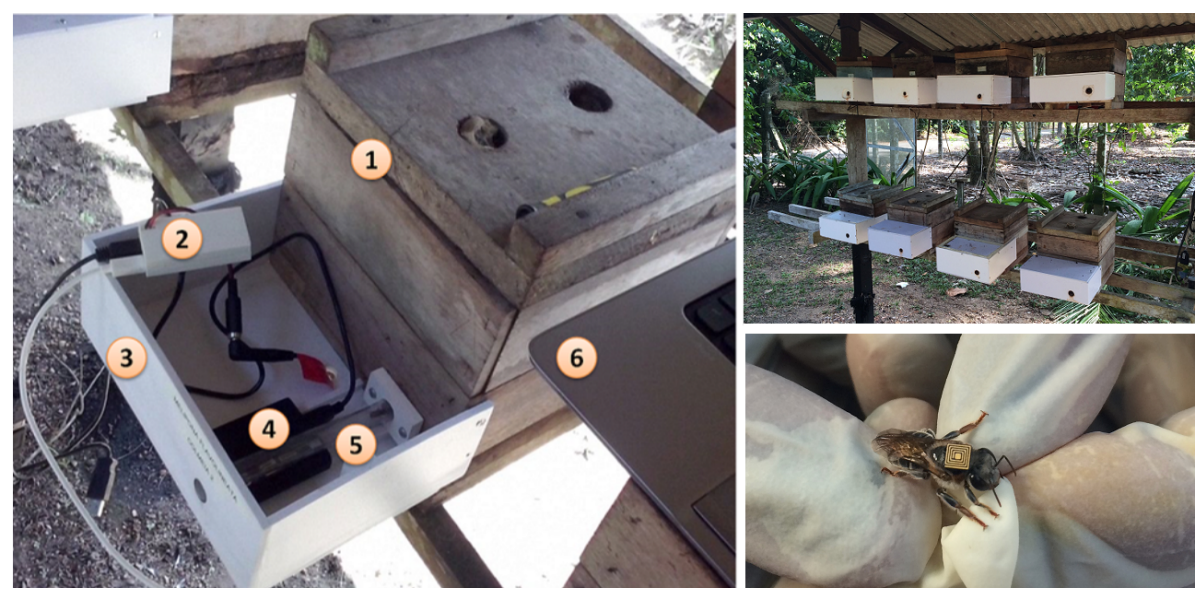

Figura 2. Sistema instrumentado para coleta de dados. Esquerda: (1) Colmeia da abelha Melipona fasciculata, (2) Intel Edison para controle do RFID e armazenamento de dados, (3) caixa de PVC para armazenamento dos itens eletrônicos, (4) leitor de antena RFID, (5) tubo plástico para a passagem das abelhas. Topo à direita: Visão geral das 8 colmeias. Inferior à direita: Abelha com etiqueta RFID anexada ao tórax. 
Para o pré-processamento, selecionamos a feature de nosso interesse: nível de atividade. No conjunto de dados bruto percebemos a presença de muitos ruídos e poucas anomalias. Assim decidimos gerar sob a base de dados original uma perturbação no conjunto de dados a fim de forçar o surgimento de mais anomalias (peça principal de investigação), gerando então anomalias sintéticas por um especialista. Assim este novo conjunto de dados foi utilizado para as próximas etapas. Os aparentes ruídos presentes na série foram mantidos para que pudéssemos verificar a efetividade dos métodos empregados para detecção de anomalia. A figura 3 ilustra a série temporal do nível de atividade de abelhas dos 5 primeiras dias de análise. Podemos notar a presença de alguns pequenos picos que sugerem a presença de ruídos. A escolha de um método que possa distinguir entre pontos ruidosos e anômalos não é uma tarefa trivial. Além disso, notamos a presença de eventos anômalos na colmeia por volta do quinto dia. Nos dias anteriores, podemos identificar padrões comportamentais dentro do nível de normalidade nas abelhas. Costumeiramente as abelhas apresentam atividade mais elevada durante o período de presença solar.

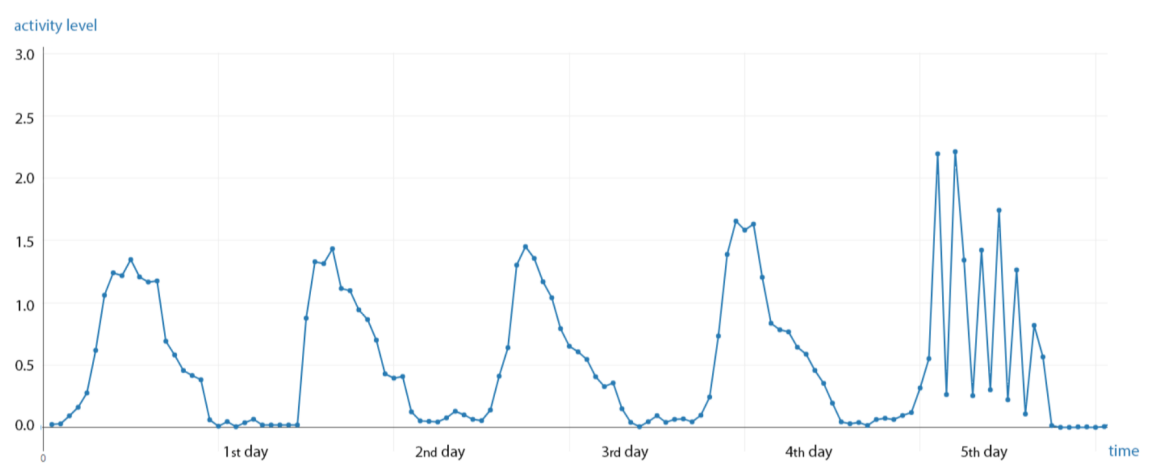

Figura 3. Serie temporal dos 5 primeiros dias de atividade das abelhas

Para a avaliação deste trabalho foram propostas três topologias de redes neurais recorrentes. A Figura 4(1) apresenta a primeira arquitetura proposta, contendo quatro camadas escondidas com um neurônio em cada. A Figura 4(2) apresenta a segunda arquitetura proposta, contendo duas camadas recorentes com quatro neurônios cada. A Figura 4(3) apresenta o terceiro modelo de arquitetura, contendo quatro neurônios dispostos em uma única camada recorrente. Nosso objetivo com essa avaliação de topologias é encontrar a rede que forneça o menor erro.

Para a implementação do algoritmo, foi utilizado o framework Keras ${ }^{1}$, o Tensorflow backend ${ }^{2}$, na linguagem Python. Os dados foram divididos em dois conjuntos (um para o treino, e outro para o teste). Do total de 733 leituras de atividade de abelha, 513 foram separadas para o treinamento, o que é equivalente a $70 \%$, as outras 220 foram utilizadas para os testes, $30 \%$. A função de ativação foi a Rectified Linear Units (ReLU) e o algoritmo otimizador, o rmsprop. O treinamento da RNN foi realizado por 500 épocas com tamanho do $b_{a t c h}{ }^{3}$ de 128. A função de custo a ser minimizada foi a entropia cruzada binária $^{4}$, visto que as classes seriam classificadas como 0 ou 1 , anomalias ou não.

\footnotetext{
${ }^{1}$ https://keras.io

${ }^{2}$ https://www.tensorflow.org/

${ }^{3}$ Quantidade de amostras utilizadas para calcular o gradiente do erro utilizado no treinamento

${ }^{4}$ Função que mede a diferença entre a saída prevista e o resultado observado
} 


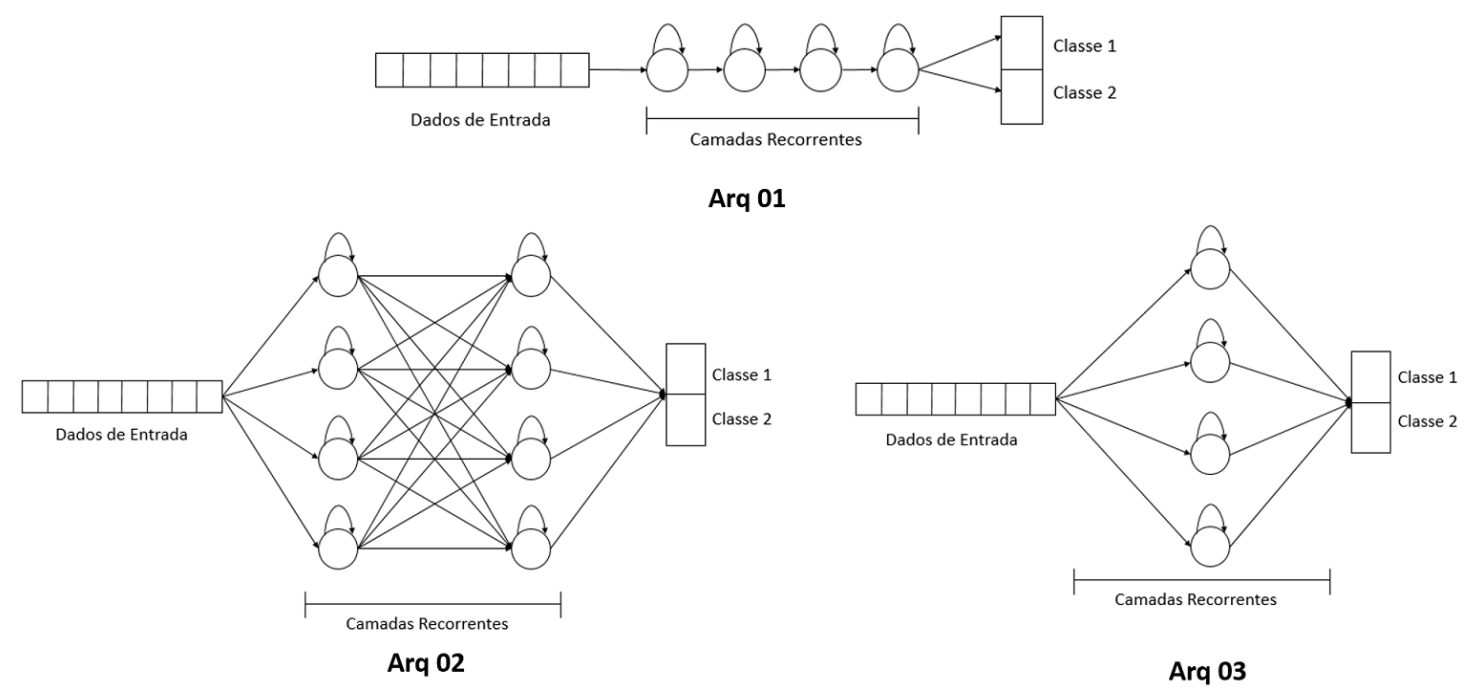

Figura 4. Modelo de arquiteturas.

\section{Resultados e Discussões}

A Figura 5 mostra o boxplot das taxas de acerto de cada RNN. Foram executadas 30 vezes cada RNN a fim de entender melhor o comportamento de cada arquitetura. Podemos ver que a arquitetura 1 apresenta restrições de aprendizado, sendo que fica estagnada. As demais arquiteturas apresentam uma variação nos resultados que são naturais devido a inicialização aleatória das RNNs. A arquitetura 2 apresenta a melhor mediana e o melhor desvio padrão dentre todas. Percebemos que a arquitetura 1 não apresentou capacidade de aprendizado, visto que os resultados se mantiveram fixos no mesmo valor $(88,18 \%)$. Esse resultado pode ser justificado pela rede responder que todos os casos são normais.

Fizemos uso de métodos estatísticos por meio do valor-p para a avaliação das arquiteturas. Usamos o método de Shapiro-Wilk para verificar a normalidade das distribuições. Caso o valor-p proveniente do método seja maior que 0.05 , as distribuições são aceitas como Gaussianas (i.e. normais). Caso o valor seja inferior a 0.05 , as distribuições não podem ser aceitas como Gaussianas, dessa forma, são consideradas não-paramétricas. Por meio do teste de Shapiro-Wilk, verificamos que as distribuições arq1 (referente à arquitetura 1) e arq3 (referente à arquitetura 3) não são aceitas como distribuições normais (valores-p inferiores a 0.05). A arq2 (referente à arquitetura 2) tem valor p próximo de 0.05, dessa forma, como as demais são não-parametricas, utilizamos o teste de Wilcoxon para comparar as distribuições. O teste de Wilcoxon mostrou p-valor inferior a 0.05 para os testes entre a melhor arquitetura candidata arq2 e as demais arq1 e arq3. Dessa forma, estatisticamente, podemos dizer que os resultados obtidos por arq2 são distintos dos demais, com $95 \%$ de confiança. Assim, podemos dizer que a arq2 é a melhor arquitetura dentre as avaliadas para resolver nosso problema.

Por fim, apresentamos os resultados obtidos por meio de Matrizes de Confusão (Tab. 6). A matriz de confusão apresenta na diagonal principal os eventos classificados corretamente, e nas outras células os eventos classificados de forma equivocada. Podemos ver que a arq2 apresentou a menor quantidade total de valores fora da diagonal principal, corroborando com a análise da taxa de acerto. 


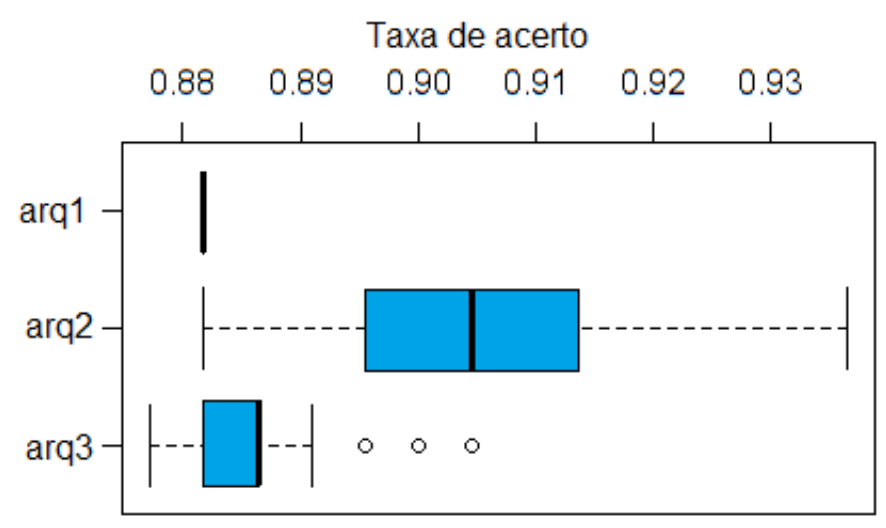

Figura 5. Boxplot dos resultados obtidos pelas arquiteturas avaliadas.

\begin{tabular}{|r|c|c|}
\cline { 2 - 3 } \multicolumn{1}{c|}{} & Normal & Anomalia \\
\hline Normal & 194 & 0 \\
\hline Anomalia & 26 & 0 \\
\hline
\end{tabular}

(a) Arquitetura 1

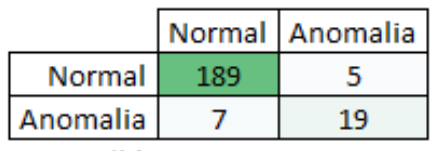

(b) Arquitetura 2

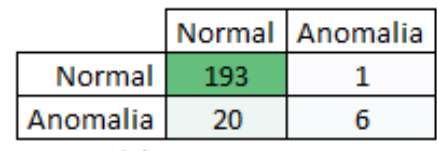

(c) Arquitetura 3

Figura 6. Matrizes de confusão para as diferentes arquiteturas. A diagonal principal apresenta os eventos classificados corretos.

\section{Conclusão}

Neste trabalho foi avaliado o uso de redes neurais recorrentes para a detecção de anomalias no comportamento de abelhas. Três arquiteturas foram avaliadas na procura de uma topologia que pudesse obter os melhores resultados. As redes neurais recorrentes foram utilizadas na intenção de avaliar se elas seriam capazes de classificar a atividade das abelhas como anômalas ou não. O estudo levou ao desenvolvimento de redes que recebem um conjunto de dados de atividade média dos insetos e é capaz de classificar este comportamento. As diferentes arquiteturas testadas mostraram que a arquitetura 2 apresentou melhor aprendizado para resolver o problema. Para trabalhos futuros seria interessante testar diferentes técnicas de detecção, assim como expandir a base de dados.

\section{Referências}

Baranauskas, J. A. and Monard, M. C. (2003). Combining symbolic classifiers from multiple inducers. Knowledge-Based Systems, 16(3):129 - 136.

Bengio, Y., Frasconi, P., Gori, M., and Soda, G. (1970). Recurrent neural networks for adaptive temporal processing.

Gama, F., Arruda, H. M., Carvalho, H. V., de Souza, P., and Pessin, G. (2017). Improving our understanding of the behavior of bees through anomaly detection techniques. In Artificial Neural Networks and Machine Learning - ICANN 2017, pages 520-527.

Hawkins, D. (1980). Identification of Outliers. Monographs on applied probability and statistics. Chapman and Hall.

Hochreiter, S. and Schmidhuber, J. (1997). Long short-term memory. Neural Comput., 9(8):1735-1780. 
Imperatriz-Fonseca, V., Contrera, F., and Kleinert, A. (2004). A meliponicultura e a iniciativa brasileira dos polinizadores. XV Congresso Brasileiro de Apicultura.

Kridi, D. S., de Carvalho, C. G. N., and Gomes, D. G. (2016). Application of wireless sensor networks for beehive monitoring and in-hive thermal patterns detection. Computers and Electronics in Agriculture, 127:221 - 235.

Lopes, M., Ferreira, J. B., and Santos, G. (2005). Abelhas sem-ferrao: a biodiversidade invisivel. Agriculturas.

Marti, L., Sanchez-Pi, N., Molina, J. M., and Garcia, A. C. B. (2015). Anomaly detection based on sensor data in petroleum industry applications. Sensors, 15(2):2774-2797.

Message, D., Teixeira, W., and Jong, D. (2012). Situação da sanidade das abelhas no brasil. In Polinizadores no Brasil: Contribuição e Perspectivas para a Biodiversidade, pages 237-356. Editora da Universidade de São Paulo.

Mitchell, T. M. (1997). Machine Learning. McGraw-Hill Science/Engineering/Math.

Nikolopoulos, C. (1997). Expert Systems: Introduction to First and Second Generation and Hybrid Knowledge Based Systems. CRC Press.

Odoux, J.-F., Aupinel, P., Gateff, S., Requier, F., Henry, M., and Bretagnolle, V. (2014). Ecobee: a tool for long-term honey bee colony monitoring at the landscape scale in west european intensive agroecosystems. Journal of Apicultural Research, 53:57-66.

P. Ballivian, J. M. P. (2008). Abelhas Nativas sem Ferrão. Oikos Ltda.

Penna, M. (2004). Rede neural artificial para deteccao de sobremortalidade atribuivel a colera no ceara. Revista de Saude Publica, 38:351 - 357.

Potts, S. G., Biesmeijer, J. C., Kremen, C., Neumann, P., Schweiger, O., and Kunin, W. E. (2010). Global pollinator declines: trends, impacts and drivers. Trends in Ecology \& Evolution, 25(6):345 - 353.

Salem, O., Liu, Y., Mehaoua, A., and Boutaba, R. (2014). Online anomaly detection in wireless body area networks for reliable healthcare monitoring. IEEE Journal of Biomedical and Health Informatics, 18(5):1541-1551.

Segatto, . A. C. and Coury, D. V. (2006). Redes neurais artificiais recorrentes aplicadas na correção de sinais distorcidos pela saturação de transformadores de corrente. Sba: Controle \& Automação Sociedade Brasileira de Automatica, 17:424 - 436.

Silva, W. P. and da Paz, J. R. L. (2012). Abelhas sem ferrao: muito mais do que uma importancia economica. Natureza on line.

Singh, K. and Upadhyaya, S. (2012). Outlier detection: Applications and techniques. International Journal of Computer Science Issues, 9(3):307-323.

Venturieri, G. C., Raiol, V., and Pereira, C. (2003). Avaliação da introdução da criação racional de melipona fasciculata (apidae: Meliponina), entre os agricultores familiares de bragança - pa, brasil. Biota Neotropica, 3:1 - 7.

Wooldridge, J. M. (2012). Introductory Econometrics: A Modern Approach.

Zacepins, A., Brusbardis, V., Meitalovs, J., and Stalidzans, E. (2015). Challenges in the development of precision beekeeping. Biosystems Engineering, 130:60 - 71 . 